\title{
Tipos de Parto e Formas de Assistência Médica em Uberaba-MG
}

\author{
Delivery and Medical Attendance Types in Uberaba-MG
}

Renato Humberto Fabri ${ }^{1}$, Eddie Fernando Candido Murta ${ }^{2}$

\begin{abstract}
RESUMO
Objetivos: estudar as reais condições da assistência médica, tipos de parto e fatores para sua indicação em Uberaba, $M G$.

Método: foram analisados os dados coletados em 7 maternidades de uma população de 4.294 puérperas que pariram no periodo de abril de 1992 a março de 1993 em Uberaba-MG. Resultados: mostrou-se que o Hospital Escola que teve a maior participação no número de partos, foi o que atendeu a população mais jovem, provavelmente a mais pobre e despreparada para a gravidez. Foi o único hospital em que as taxas de cesariana foram próximos àqueles aceitáveis pela OMS. Em Uberaba o tipo de assistência médica foi predominantemente remunerada pelo SUS ficando os convênios e atendimentos particulares com proporção bem inferior. Verificou-se também que a frequência de cesariana se elevou com a idade e tipo de assistência médica, e o grupo de conveniadas e particulares ficaram com o maior número de cesarianas.

Conclusões: pode-se talvez especular que o fator social esteja interferindo na indicação do tipo parto.
\end{abstract}

PALAVRAS-CHAVE: Gestação: resolução. Custos em saúde. Parto. Cesariana.

\section{Introdução}

Fatores ligados à assistência médica, à precária educação e orientação às gestantes, assim como causas de natureza econômica, têm, provavelmente, proporcionado elevado número de parto cesariano em detrimento do parto normal.

\footnotetext{
${ }^{1}$ Departamento de Medicina Social da FMTM

${ }^{2}$ Disciplina de Ginecologia e Obstetrícia da FMTM

Faculdade de Medicina do Triângulo Mineiro - Departamento de Medicina Social e Disciplina de Ginecologia e Obstetrícia Correspondência:

Renato Humberto Fabri

Departamento de Medicina Social

Rua Getúlio Guaritá, s/n ${ }^{\circ}$ - Abadia

38025-440 - Uberaba - MG
}

Gentile de $\mathrm{Melo}^{9}$, em seu estudo já mostrava a influência do poder econômico na escolha do tipo de parto, com a escolha da data de nascimento e, muitas vezes com a esterilização cirúrgica. Assim, as proporções de cesarianas em hospitais conveniados ao INPS, oscilaram de 9,7 a 14\%, ao passo que nos hospitais assistenciais os valores eram semelhantes ao dos Estados Unidos da América (7\%).

Associado a esses fatores, o progresso científico e o aprimoramento técnico têm facilitado o aumento de número de partos cirúrgicos ${ }^{14}$, e a indicação do tipo de parto, parece estar associada ao tipo de assistência médica.

Janowitz et $\mathrm{al}^{10}$, ao analisarem tipos de partos em 9 hospitais no sul do Brasil, encontraram um 
predomínio de partos cesarianos entre pacientes particulares e conveniados em relação às demais. Do mesmo modo Siqueira et al. ${ }^{13}$, em São Paulo, ao analisarem puérperas de duas maternidades de níveis sócio-econômicos diferentes, também encontraram uma maior proporção de parto cesariano nas maternidades particulares.

Em 1986 entre 349 mulheres que deram à luz num Serviço de Obstetrícia da Grande São Paulo, Lopez et al. ${ }^{11}$ verificaram que a proporção de cesarianas foi mais alta entre puérperas que tiveram um pequeno número de consultas no prénatal. Relataram uma taxa de cesariana de $20,3 \%$, que foi considerada baixa se comparada com aquela do Estado de São Paulo que é de $43,7 \%$, que por sua vez é um valor significativamente mais elevado do que os preconizados pelo OMS (10 a $15 \%)^{12}$.

Beleza Filho et al. ${ }^{2}$ verificaram em Florianópolis 30,5 \% de parto cesariano, Lopez et al. ${ }^{11} 34,4$ \%, Bettiol $^{3}$ em Ribeirão Preto 30,4 \% e Fabri $^{6}$ em Uberaba, encontrou 48,7\%. Aranha ${ }^{1}$, em estudo em maternidade privada no Rio de Janeiro, mostrou que $78 \%$ dos partos foram cesariana, Camano et al. ${ }^{4}$ na Escola Paulista de Medicina verificaram entre 1975 a 1981 uma elevação das taxas de cesariana de $25,5 \%$ para $37,9 \%$. Costa ${ }^{5}$, ao estudar a incidência do parto cesariano, verificou que este vem se elevando progressivamente nos últimos 20 anos.

Diante da importância do assunto em análise, propusemo-nos a realizar em Uberaba um estudo com o objetivo de averiguar as reais condições da assistência médica e tipos de partos, assim como procurar os fatores intervenientes na sua indicação.

\section{Material e Métodos}

O presente trabalho foi desenvolvido com visitas diárias às maternidades de Uberaba no periodo de abril de 1992 a abril de 1993. O mesmo faz parte de um projeto bem mais amplo sobre Saúde Materno-Infantil que incluiu itens relativos aos pais, à renda, à gestação, ao parto, e ao tipo de atenção médica.

O preenchimento dos questionários foi realizado com entrevistas a puérperas que deram à luz a um ou mais nativivos e que estivessem de acordo com os propósitos do estudo. Por questões éticas não foram entrevistadas as mães que não deram seu consentimento ou se o recém-nato não estivesse em boas condições de saúde. Em caso de alta precoce, a entrevista era realizada no domicílio até 15 dias após o parto.
Para que fosse possivel seu desenvolvimento, a equipe foi constituída de estudantes do Curso de Enfermagem da Faculdade de Medicina do Triângulo Mineiro (FMTM). No período de treinamento, fez-se a aplicação dos questionários durante 15 dias nos hospitais envolvidos (estudo piloto) com objetivo de se criar condições de padronização das entrevistas e homogeneidade nas condições do trabalho. As entrevistadoras se revezavam nos hospitais, sendo que uma ficou encarregada do controle do recebimento dos questionários, correção do preenchimento e da ordenação do material para codificação. Para que o projeto pudesse ter um desenvolvimento adequado, o responsável além do acompanhamento diário, reunia-se com todo o grupo a cada 15 dias com o intuito de discutir e propor soluções para os problemas surgidos. Completado o questionário, esse era revisado e encaminhado para aferição sendo utilizado um manual previamente elaborado.

Codificadas as informações, essas foram digitadas e transferidas para discos magnéticos sendo posteriormente conferidas por outro digitador (método de dupla digitação) e só então analisadas.

\section{Resultados}

Durante o desenvolvimento da pesquisa, 4.294 mulheres pariram em hospitais de Uberaba, sendo que $85 \%$ foram procedentes da zona urbana de Uberaba e de outras localidades.

A distribuição se encontra na Tabela 1. Nesta tabela, verifica-se que a grande maioria dos partos $(37,8 \%)$ ocorreu no Hospital Escola (Hospital A), valor que variou de 2,5 à 5 vezes o número encontrado nos outros hospitais. Nota-se que a distribuição por idade não é homogênea entre os hospitais, sendo que o Hospital Escola teve a maior proporção de puérperas jovens (28\%). Ao contrário, o Hospital B, teve uma clientela com idade mais elevada. Por problemas referentes à "recusa da entrevista" e/ou informações com código "ignorado" ou informações incompativeis com os dados fornecidos, o número total de casos poderá variar nas tabelas.

Na Tabela 2 verifica-se que no Hospital Escola a proporção de partos cesarianos se eleva monotonicamente com o aumento da idade das pacientes, ao passo que nos hospitais da rede privada o comportamento é semelhante, mas as proporções são bem mais elevadas.

Admitindo-se que a indicação do parto cirúrgico possa estar associado à duração da gestação, procurou-se com os dados expostos na 
Tabela 3, uma possivel explicação. No Hospital Escola, a proporção de cesariana é maior no grupo com idade gestacional inferior a 38 semanas, ao passo que nos outros hospitais essa proporção é praticamente a mesma nos três grupos etários, e bem superiores àqueles do Hospital Escola.

Os recursos gestacionais utilizados pelas puéperas foram divididos em três categorias segundo o tipo de parto e hospitais, conforme exposto na Tabela 4. A maioria das internações foram pagas pelo SUS (74,3\%), seguido com grande diferença pelos convênios (14,6\%) e pelas de caráter particular $(11,2 \%)$. Foi na categoria dos SUS em que ocorreu a maior proporção de partos normais $(58,7 \%)$, ao passo que para os convênios e particulares estes valores foram até 4 vezes menores. Os dados nos mostram que o Hospital Escola teve a menor proporção de cesariana em relação aos demais hospitais, e que a indicação cirúrgica é significativamente maior entre as pacientes que utilizaram os convênios ou particulares.

Tabela 1 - Distribuição das pacientes que pariram em Uberaba, no período de estudo, segundo idade e hospital onde ocorreu o parto.

\section{Idade}

\begin{tabular}{lrrrrrrrr} 
Hospitais & \multicolumn{2}{c}{$<\mathbf{2 0}$} & \multicolumn{2}{c}{$\mathbf{2 0} \mathbf{- 3 0}$} & \multicolumn{2}{c}{$>$ 30 } & \multicolumn{2}{c}{ Total } \\
\cline { 2 - 10 } & $\mathbf{n}$ & $\mathbf{\%}$ & $\mathbf{n}$ & $\mathbf{\%}$ & $\mathbf{n}$ & $\mathbf{\%}$ & $\mathbf{n}$ & $\mathbf{\%}$ \\
\hline A & 458 & 28,2 & 871 & 53,6 & 296 & 18,2 & 1.625 & 37,8 \\
B & 14 & 4,0 & 196 & 56,8 & 135 & 39,1 & 345 & 8,0 \\
C & 103 & 18,3 & 364 & 64,6 & 96 & 17,0 & 563 & 13,1 \\
D & 47 & 10,6 & 292 & 66,1 & 102 & 23,1 & 441 & 10,3 \\
E & 49 & 14,6 & 213 & 66,3 & 74 & 22,0 & 336 & 7,8 \\
F & 38 & 12,0 & 207 & 65,3 & 72 & 22,7 & 317 & 7,4 \\
G & 99 & 15,2 & 393 & 60,4 & 158 & 24,3 & 650 & 15,1 \\
H & 06 & 35,3 & 09 & 52,9 & 02 & 11,8 & 17 & 0,4 \\
\hline TOTAL & 814 & 18,9 & 2.545 & 59,3 & 935 & 21,8 & 4.294 & 100,0
\end{tabular}

Tabela 2 - Distribuição das puérperas estudadas que pariram em Uberaba, segundo idade, tipo de parto e hospitais.

Idade/Tipo de Parto

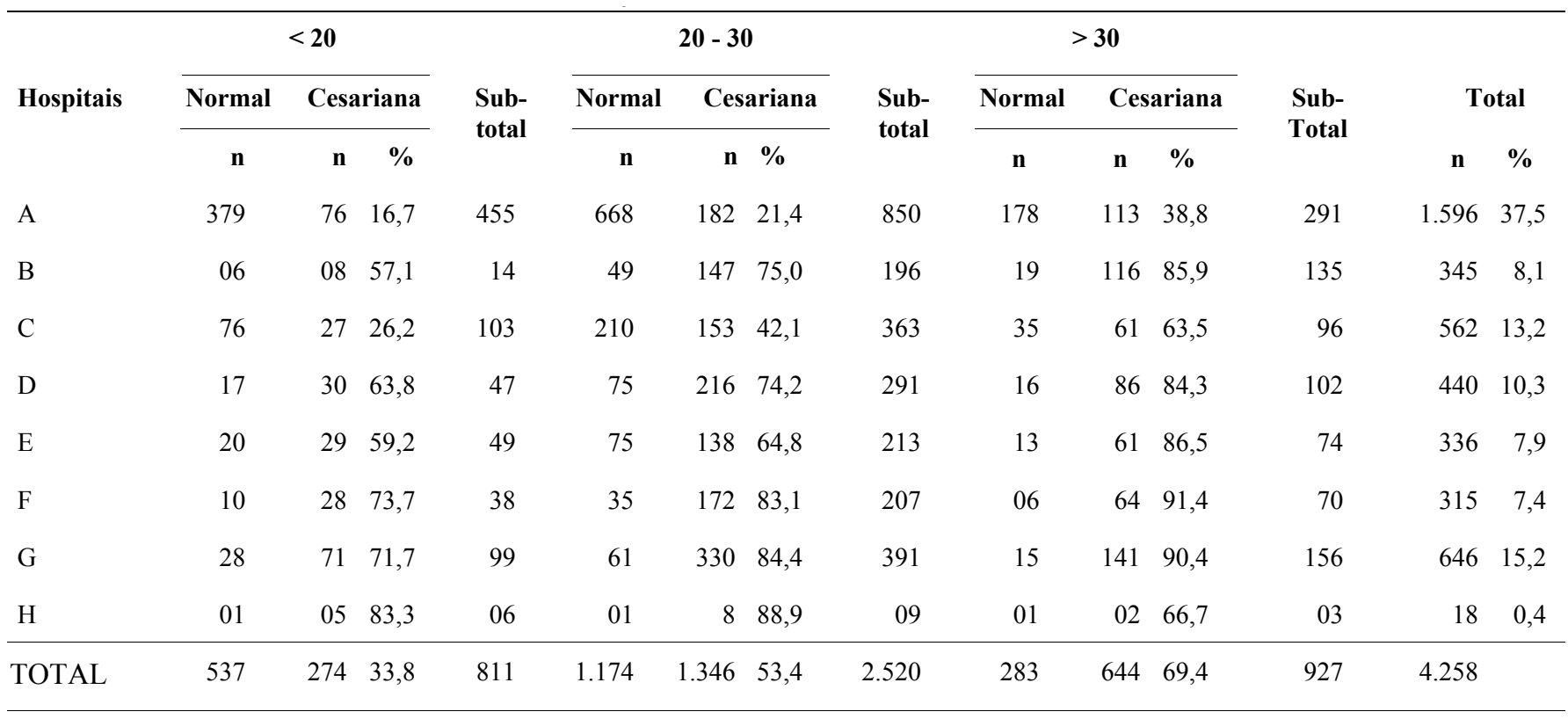


Tabela 3 - Distribuição das puérperas estudadas, segundo o hospital, tempo de duração da gestação e tipo de parto.

Duração da Gestação/Tipo de parto

\begin{tabular}{|c|c|c|c|c|c|c|c|c|c|c|c|c|c|}
\hline \multirow[t]{2}{*}{ Hospital } & \multicolumn{3}{|c|}{$<38$ semanas } & & \multicolumn{3}{|c|}{38 - 42 semanas } & \multicolumn{4}{|c|}{$>42$ semanas } & \multirow{2}{*}{$\begin{array}{l}\text { Sub- } \\
\text { total }\end{array}$} & \multirow{2}{*}{ Total } \\
\hline & $\frac{\text { Normal }}{n}$ & \multicolumn{2}{|c|}{ Cesariana } & $\begin{array}{l}\text { Sub- } \\
\text { total }\end{array}$ & $\begin{array}{c}\text { Normal } \\
\mathbf{n}\end{array}$ & \multicolumn{2}{|c|}{ Cesariana } & $\begin{array}{l}\text { Sub- } \\
\text { total }\end{array}$ & $\begin{array}{c}\text { Normal } \\
\mathbf{n}\end{array}$ & \multicolumn{2}{|c|}{ Cesariana } & & \\
\hline A & 203 & 70 & 25,6 & 273 & 554 & 168 & 23,3 & 722 & 482 & 133 & 21,6 & 615 & 1610 \\
\hline TOTAL & 353 & 431 & 54,9 & 784 & 982 & 1.311 & 57,8 & 2.293 & 673 & 522 & 43,7 & 1.195 & 4.272 \\
\hline
\end{tabular}

Tabela 4 - Distribuição das puérperas que pariram em Uberaba, segundo a categoria de internação, tipo de parto e hospital.

\begin{tabular}{|c|c|c|c|c|c|c|c|c|c|c|c|c|c|}
\hline \multirow{4}{*}{ Hospital } & \multicolumn{13}{|c|}{ Categoria de internação / Tipo de parto } \\
\hline & \multicolumn{3}{|c|}{ SUS } & \multirow{3}{*}{$\begin{array}{l}\text { Sub- } \\
\text { total }\end{array}$} & \multicolumn{3}{|c|}{ Convênio } & \multirow{3}{*}{$\begin{array}{l}\text { Sub- } \\
\text { total }\end{array}$} & \multicolumn{3}{|c|}{ Particular } & \multirow{3}{*}{$\begin{array}{l}\text { Sub- } \\
\text { total }\end{array}$} & \multirow{3}{*}{ Total } \\
\hline & \multirow{2}{*}{$\begin{array}{c}\text { Normal } \\
\text { n }\end{array}$} & \multicolumn{2}{|c|}{ Cesariana } & & Normal & \multicolumn{2}{|c|}{ Cesariana } & & Normal & \multicolumn{2}{|c|}{ Cesariana } & & \\
\hline & & $\mathbf{n}$ & $\%$ & & $\mathbf{n}$ & $\mathbf{n}$ & $\%$ & & $\mathbf{n}$ & $\mathbf{n}$ & $\%$ & & \\
\hline A & 1.201 & 362 & 23,1 & 1.563 & 0 & 0 & 0 & 0 & 0 & 0 & 0 & 0 & 1563 \\
\hline B & 47 & 66 & 58,4 & 113 & 20 & 130 & 86,7 & 150 & 7 & 72 & 91,1 & 79 & 342 \\
\hline $\mathrm{C}$ & 271 & 141 & 34,2 & 412 & 18 & 51 & 73,9 & 69 & 24 & 43 & 64,1 & 07 & 548 \\
\hline $\mathrm{D}$ & 91 & 131 & 59,0 & 222 & 12 & 154 & 92,8 & 166 & 3 & 46 & 93,9 & 49 & 437 \\
\hline $\mathrm{E}$ & 88 & 101 & 53,4 & 189 & 09 & 50 & 84,7 & 59 & 7 & 71 & 91,0 & 78 & 326 \\
\hline $\mathrm{F}$ & 27 & 112 & 80,6 & 139 & 08 & 77 & 90,6 & 85 & 15 & 71 & 82,5 & 86 & 310 \\
\hline $\mathrm{G}$ & 93 & 355 & 79,2 & 448 & 03 & 76 & 96,2 & 79 & 07 & 101 & 93,5 & 108 & 635 \\
\hline $\mathrm{H}$ & 03 & 14 & 82,3 & 17 & 0 & 0 & 0 & 0 & 0 & 0 & 0 & 0 & 17 \\
\hline TOTAL & 1.821 & 1.282 & & 3.103 & 70 & 538 & & 608 & 63 & 404 & & 467 & 4.178 \\
\hline$\%$ & 58,7 & 41,3 & & 74,3 & 11,3 & 88,5 & & 14,6 & 13,5 & 86,5 & & 11,2 & \\
\hline
\end{tabular}

\section{Discussão}

Os dados da pesquisa mostram que a grande maioria dos partos de Uberaba ocorreu no Hospital Escola $(37,8 \%)$ e que este ficou com a população mais jovem $(28,2 \%$ das pacientes com menos de 20 anos).

A proporção de cesariana no Hospital Escola é bem inferior em relação aos outros hospitais, com taxa de $23,2 \%$ das cesarianas, enquanto que o municipio apresenta uma taxa de $53,2 \%$.

A Tabela 3 mostra que no Hospital Escola as taxas de cesarianas são mais elevadas entre as pacientes com gestação menor de 38 semanas do que nos demais grupos. Para os outros hospitais, os níveis de cesariana são muito elevados e pouco variam com a idade gestacional. Isto nos faz pensar que a duração da gestação não é um fator importante na indicação de cesariana para os hospitais particulares.

As taxas elevadas de cesariana poderiam ser explicadas se nos hospitais privados ocorresse uma maior proporção de partos com gestação de menor duração. Este fato não é verdadeiro, pois o Hospital Escola teve as menores proporções de 
cesariana nos grupos com menos de 38 e nos maiores de 42 semanas de gestação, ficando assim, dificil tal explicação. Uma possivel justificativa seria a realização de outros procedimentos simultaneamente ao parto (exemplo: laqueadura), ou até mesmo a disponibilidade de recursos que são poucos entre as puérperas jovens.

Em Uberaba, o SUS ficou responsável por $74,3 \%$ da assistência ao parto. O Hospital Escola teve a menor proporção de cesariana $(23,1 \%)$. Quando a categoria de internação passa a convênio ou particular, a elevação das taxas é mais evidente, pois aqui estão as parturientes que têm melhor condição sócio-econômica. Estudos de Janowitz et al. ${ }^{10}$ em hospitais dos estados de São Paulo e Rio de Janeiro mostram que em quase todos os hospitais as taxas de cesariana aumentaram ao longo da classificação: indigentes ( $25 \%$ ou menos), segurados $(40 \%)$ e particulares $(75 \%)$.

Siqueira et al. ${ }^{13}$, em São Paulo, verificaram que a taxa de cesariana elevou-se 4 vezes quando comparando um hospital assistencial com um particular (10\% e 39,9\%, respectivamente).

Assim, os dados deste estudo permitiram mostrar que:

a. O Hospital Escola teve maior participação no número de partos e foi o que abrigou a população mais jovem;

b. Foi o único hospital em que as taxas de cesariana foram próximos dos valores aceitáveis pela OMS;

c. Em Uberaba, o tipo de assistência médica foi predominantemente pelo SUS, ficando os convênios e particulares com proporção bem inferior;

d. A freqüência de cesariana se elevou com a idade e tipo de assistência médica, sendo que entre as puérperas conveniadas e particulares a proporção aumenta de forma inexplicável.

Esses dados não estão em total desacordo com aqueles encontrados na literatura ${ }^{4,7,8}$, pois há em todo o mundo um alargamento das indicações das cesarianas, com conseqüente elevação de sua incidência.

As elevadas proporções de partos nos hospitais de Uberaba não encontraram respaldo científico se comparados àqueles encontrados no Hospital Escola. Pelo contrário, os dados parecem indicar que uma expressiva proporção desse tipo de parto é realizada por motivos outros que não só as indicações médicas, mas provavelmente ligada a fatores econômicos, culturais e sociais.

\section{SUMMARY}

Purpose: to study the actual conditions of medical assistance and types of delivery and factors contributing to their indication in Uberaba, $M G$.

Method: the data of 4,294 puerperas who gave birth in the period from April 15, 1992 to April 14, 1993 in 7 maternities in Uberaba were studied.

Results: it was seen that the Teaching Hospital had a greater participation in deliveries attending the younger population, probably the poorest and most unprepared regarding pregnancy. It was the only Hospital in which cesarean section rates were near those accepted by the WHO. Medical assistance in Uberaba was predominantly through Social Security (SUS), private health insurance and physicians representing a lower proportion. It was also verified that cesarean section frequency increased with age and type of medical assistance and the groups with private coverage presented a higher number of cesarean sections.

Conclusion: it may be perhaps justified to consider the social factor as interfering with the indication of type of delivery.

KEY WORDS: Pregnancy outcome. Health costs. Delivery. Cesarean section.

\section{Referências}

1. Aranha EG. Cesarianas, indicações atuais. J Cons Fed Med 1997; 81:3.

2. Beleza Filho AAL, Balotto LC, Saab Neto JÁ, Garcia WZ. Gestação na adolescência. J Bras Ginecol 1984; $94: 271-4$

3. Bettiol H. Saúde perinatal em Ribeirão Preto: estudo de algumas variáveis sociais e biológicas no perfil reprodutivo de mães adolescentes. Dissertação de Mestrado. Faculdade de Medicina de Ribeirão Preto - USP 1990. p.166.

4. Camano L, Vigorito NM, Mattar R. Aumento da freqüência da cesariana. Análise de percentagem verificada na Escola Paulista de Medicina. J Bras Ginecol 1984; 94: 407-10.

5. Costa PL. Aspectos atuais da operação cesariana: incidência. Femina 1981; 9:61-5.

6. Fabri RH. Estudo de algumas características da gestação na adolescência, Uberaba-MG. Tese de Doutorado. Faculdade de Medicina de Ribeirão Preto - USP - 1996. p. 87.

7. Fiori RM, Fiori HH, Hentschel H. Mortalidade perinatal no Rio Grande do Sul - Estudo comparativo de 16 maternidades. J Pediatr 1989; 65: 72-85. 
8. Gardonyi Carvalheiro CD. Padrões de atendimento ao parto no município de Ribeirão Preto, São Paulo, Brasil. Bol OPAS 1978; 85:239-49.

9. Gentile MC. Privatizações e produtividade dos serviços de saúde: pesquisa setorial sobre a incidência de cesariana. R Paul Hosp 1982; 17:3-11.

10. Janowitz B, Nakamura MS, Lins FE, Brown ML, Ceopton D. Cesarean section in Brazil. Soc Sci Med 1982; 16: 19-25.

11. Lopez AFV, Schor N, Siqueira AAF. Gravidez na adolescência: estudo comparativo. R Saúde Públ São Paulo 1989; 23:473-7.
12. Ministério da Saúde. Assistência integral à saúde da mulher: bases de ações programáticas. Brasília, Centro de Documentação do Ministério da Saúde 1984.

13. Siqueira AAF, Areno FB, Almeida PAM, Tanaka, ACA. Relação entre peso ao nascer, sexo do recém-nato e tipo de parto. Rev Saúde Públ São Paulo 1981; 15:283-90.

14. Williams RL, Hawes WE. Cesarean section, fetal monitoring and perinatal mortality in California. Am J Public Health 1979; 69: 864-70.

\section{8음 CONGRESSO BRASILEIRO DE GINECOLOGIA E OBSTETRÍCIA}

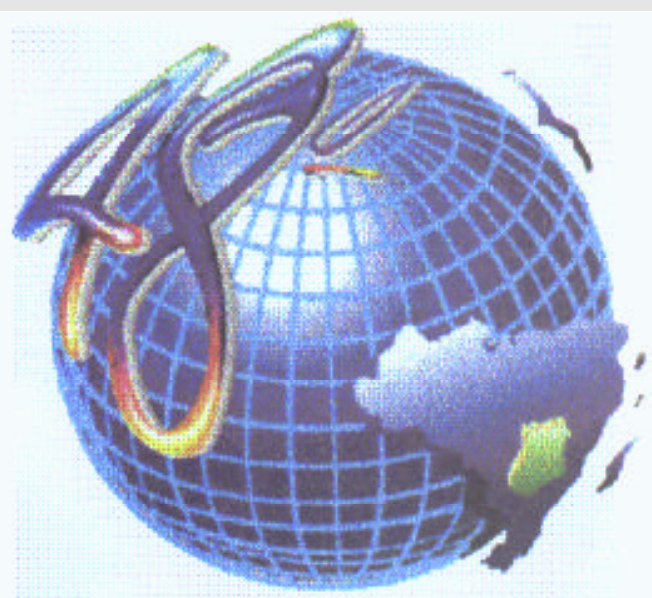

\section{A 27 DE NOVEMBRO DE 1999}

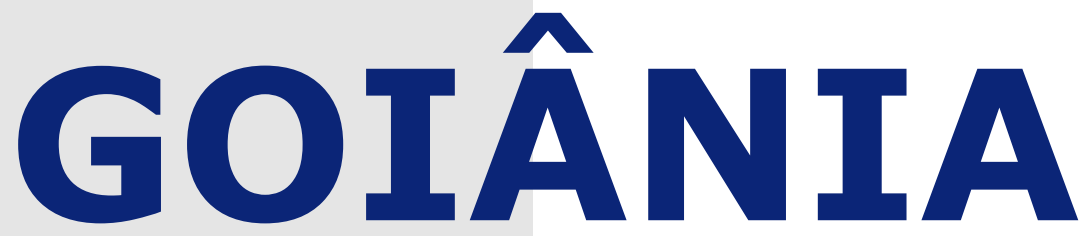

INFORMAÇÕES:

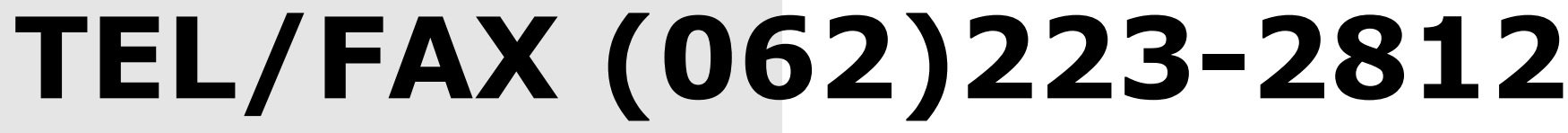

